\title{
Performance and Flatness of a Multiple-Cathode, Rectangular Ion Thruster Discharge Chamber
}

\author{
Joshua L. Rovey* and Alec D. Gallimore ${ }^{\dagger}$ \\ University of Michigan, Ann Arbor, Michigan 48109 \\ DOI: $10.2514 / 1.21325$
}

\begin{abstract}
Results from the extended life test of the Deep Space One flight spare ion engine show that enlargement of the discharge cathode assembly orifice due to erosion of the orifice by ion bombardment limits the throughput of the ion thruster, and therefore limits its operational lifetime to approximately three years. Future deep-space missions will require significantly longer operational lifetime, perhaps as long as 7-14 years. In an effort to increase lifetime, an ion thruster discharge chamber designed for operation with multiple discharge cathode assemblies was investigated. The multiple-cathode discharge chamber approach attempts to increase lifetime by operating three discharge cathode assemblies sequentially. Simulated ion thruster operation of the multiple-cathode discharge chamber with the active discharge cathode assembly located on centerline and off centerline for a variety of magnetic field configurations was accomplished. Results indicate that the configuration with permanent magnets and $\mathbf{0} \mathrm{A}$ electromagnet current provided the best performance and flatness with optimum values of $194 \pm 6 \mathrm{~W} / \mathrm{A}$ at $0.89 \pm 0.03$ propellant efficiency and $0.55 \pm 0.02$, respectively. Finally, operation of the dormant cathodes with propellant flow is suggested to reduce preoperation erosion of those units.
\end{abstract}

\section{Nomenclature}

$I_{\text {emag }}=$ electromagnet current, $\mathrm{A}$

$J_{b}=$ beam current, A (current leaving MCDC during simulated operation)

$J_{d} \quad=$ discharge current, $\mathrm{A}$

$J_{g} \quad=$ grid-plane ion current, $\mathrm{A}$

$J_{S}=$ ion collection grid current, $\mathrm{A}$

$\dot{\dot{m}} \quad=$ reduced mass flow rate, $\mathrm{A}$

$V_{d}=$ discharge voltage, $\mathrm{V}$

$\varepsilon_{b}=$ ion production cost, $\mathrm{W} / \mathrm{A}$

$\eta_{\text {ud }}=$ propellant utilization efficiency, $\%$

$\phi_{i}=$ high-voltage optics ion transparency $(\sim 70 \%)$

$\phi_{i}=$ ion collection grid ion transparency

\section{Introduction}

ION thrusters are high-efficiency, high-specific impulse propulsion systems that are being proposed as the primary propulsion source for a variety of advanced, deep-space missions. An ion thruster that can satisfy the mission requirements will require long life, high power, and high-specific impulse. In fact, an ion thruster may be required to operate continuously for as long as 7 14 years [1-3]. Results from the extended life test (ELT) of the flight spare Deep Space One NASA solar electric propulsion technology and applications readiness (NSTAR) ion engine show that ion bombardment erosion of a molybdenum (Mo) keeper discharge cathode assembly (DCA) limits the operational lifetime of the ion

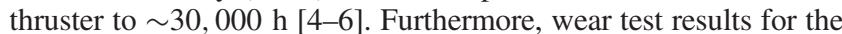
NASA evolutionary xenon thruster (NEXT) with a Mo keeper DCA show wear profiles similar to the NSTAR thruster [7], suggesting it

Presented as Paper 4407 at the 41st Joint Propulsion Conference, Tucson, AZ, 10-13 July 2005; received 22 November 2005; accepted for publication 16 April 2006. Copyright $\odot 2006$ by Joshua L. Rovey. Published by the American Institute of Aeronautics and Astronautics, Inc., with permission. Copies of this paper may be made for personal or internal use, on condition that the copier pay the $\$ 10.00$ per-copy fee to the Copyright Clearance Center, Inc., 222 Rosewood Drive, Danvers, MA 01923; include the code $\$ 10.00$ in correspondence with the CCC.

${ }^{*}$ Graduate Student Researcher, Plasmadynamics and Electric Propulsion Laboratory, Department of Aerospace Engineering, 1060 FXB Building, 1320 Beal Avenue. Student Member AIAA.

${ }^{\dagger}$ Arthur F. Thurnau Professor, Plasmadynamics and Electric Propulsion Laboratory, Department of Aerospace Engineering, 3037 FXB Building, 1320 Beal Avenue. Associate Fellow AIAA. may also be limited in life due to ion bombardment erosion. Based on these results, one can conclude that contemporary ion thrusters using a single, Mo keeper DCA are incapable of satisfying future mission requirements due to plasma ion bombardment of the DCA [5]. As a result, the NEXT, high-power electric propulsion (HiPEP) thruster, and nuclear electric xenon ion system (NEXIS) thruster are using carbon graphite keepers to increase thruster throughput and operational lifetime [7-9]. However, Mo keeper DCAs may still satisfy mission requirements if multiple DCAs are used.

Other DCA failure mechanisms can also occur after prolonged operation. Specifically, depletion of the barium in the insert, brought on by simple barium diffusion and subsequent evaporation, or the formation of tungstates that tie up the barium. Because of such phenomena, a single ion thruster hollow cathode may not be sufficient for missions requiring over four years of continuous thruster operation [10].

To increase thruster lifetime, an ion thruster with three sequentially operated DCAs was investigated. With this approach, a new DCA is ignited when the previous one fails. Ideally, this will increase thruster discharge lifetime threefold, making longer mission times a possibility. The following sections describe the design of the multiple-cathode discharge chamber (MCDC), apparatus used to operate the MCDC as a simulated ion thruster [11], results, analyses, and conclusions.

\section{Multiple-Cathode Discharge Chamber Design}

In an effort to extend the lifetime of an ion thruster, a HiPEPderivative ion thruster that operates three DCAs sequentially was designed. Investigation of various MCDC designs was initiated using the HiPEP engine baseline dimensions [12] and the NEXT magnetic field topology. Several magnetic field designs were investigated and evaluated based on a set list of criteria. Each design was solved numerically using the 3-D magnetostatic code $\mathrm{MagNet}^{\mathrm{TM}} 6.0$ [13], which provides the magnetic field topology for each design. The following sections describe the criteria used to determine the selected discharge chamber magnetic field and the selected MCDC.

\section{A. Design Criteria}

To facilitate the operation of three DCAs, a discharge chamber must have a magnetic field that fulfills certain criteria. More specifically, the magnetic field must exhibit similar characteristics at each of the three DCA locations inside the chamber. Because a DCA 
identical to the NEXT DCA was used in this investigation and the NEXT is a state-of-the-art ion thruster, the design goal was to place each DCA in a magnetic field environment similar to the NEXT DCA. Accomplishing this objective assured that each DCA will function in a manner similar to the proven electron source of the NEXT.

The selected MCDC design was required to exhibit the following characteristics. First, the magnetic field lines at the exit of the cathodes are uniform and parallel to the cathode axis. This allows exiting electrons to spiral away along the field lines $[14,15]$. Second, the exit of the cathode is located downstream of the peak magnetic field strength. This feature assists electrons in falling away from the cathode and out into the discharge chamber. Placing the cathode upstream of the peak field strength may lead to a mirroring effect, which can cause expelled electrons to become trapped near the cathode $[14,15]$. Third, the magnitude of the magnetic field contours at the cathode exit plane are equal to the NEXT magnetic field to facilitate the residence time necessary for propellant ionization. Finally, the permanent magnet ring spacing was designed such that the intercusp field strength is similar to NEXT.

\section{B. Selected MCDC}

\section{Anode}

The selected MCDC has a rectangular shape and a rectangular ion extraction area of $3600 \mathrm{~cm}^{2}$. Nonmagnetic stainless steel sheet metal was used to construct the backplate and the rectangular shell. Four corner brackets were welded to the sheet metal to form the rectangular shell and the backplate was bolted to the shell to form the five-sided anode, i.e., a cuboid with an open face. Three holes centered in the backplate were spaced a few centimeters linearly apart for placement of the DCAs.

\section{Permanent Magnets}

Samarium Cobalt permanent magnets were used to form the baseline magnetic circuit. A circular magnet ring was mounted to the backplate at each of the three DCA locations. Two concentric rectangular rings surrounded the three DCAs and two more rectangular rings were located on the anode walls.

\section{Electromagnet}

In addition to the baseline all-permanent-magnet magnetic circuit, an electromagnet was used to augment and change the magnetic field. A coil with 280 turns of 15-gauge magnet wire was wrapped in a double-conductor configuration around a rectangular aluminum bobbin and placed inside a magnetic iron channel. The use of two conductors reduces the length of the wire and subsequently its resistance so that a lower voltage can be used to drive the electromagnet current. The magnetic channel increases the efficiency of the electromagnet by directing the flux, thus the electromagnet requires fewer turns and less current than if operated without the magnetic iron. The near-DCA magnetic field was adjusted by mounting the electromagnet externally to the backplate and supplying a current within the range of \pm 10 A. Negative current decreases the near-DCA magnetic field and positive current increases it. With the electromagnet attached, the DCA exit-plane magnetic field strength can be adjusted from $15 \mathrm{G}$ to over $100 \mathrm{G}$. To recover the baseline all-permanent-magnet configuration, the electromagnet must be operated at $-5 \mathrm{~A}$. This result is due to the presence of the magnetic iron material, which increases the allpermanent-magnet magnetic field, even without electromagnet current.

\section{Magnetic Field Configurations}

Although an infinite number of magnetic field configurations are possible because the electromagnet can be set at any desired current level, only seven were investigated in this work. Five magnetic field configurations were studied by setting the electromagnet current at $\pm 10 \mathrm{~A}, \pm 5 \mathrm{~A}$, and $0 \mathrm{~A}$. A sixth configuration enclosed the $50 \mathrm{G}$ contour within the MCDC by attaching the electromagnet (operated

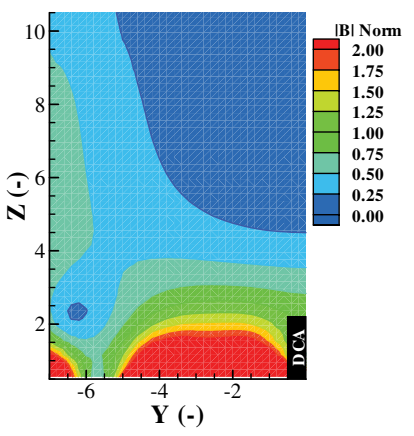

a) 50 G contour enclosed
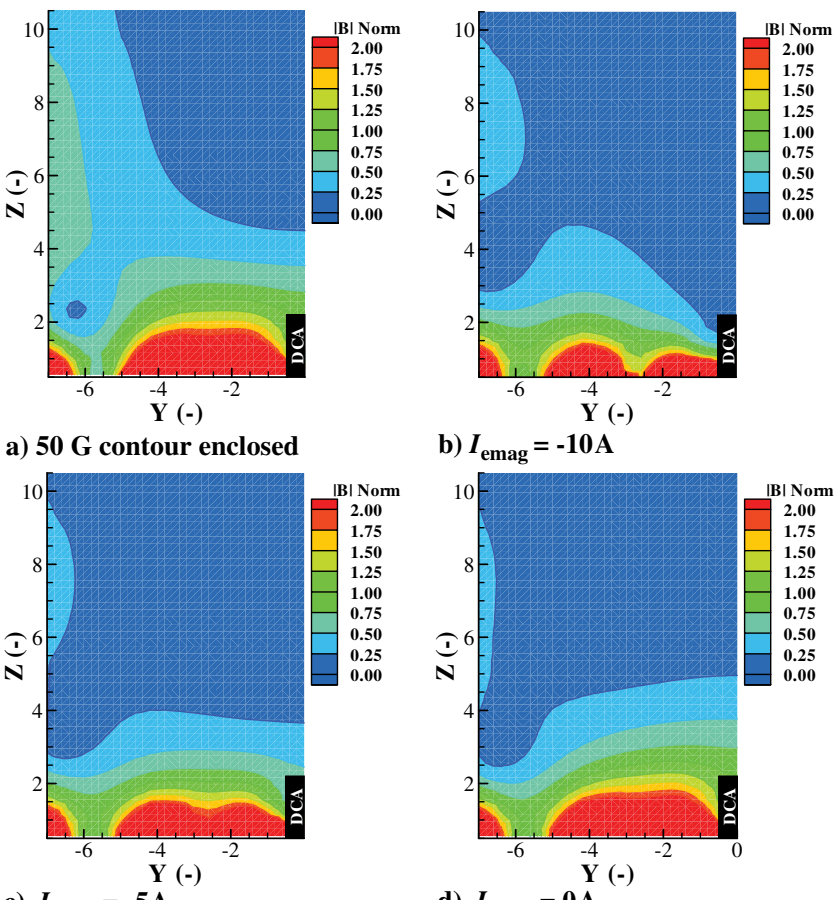

c) $I_{\mathrm{emag}}=-\mathbf{5 A}$

b) $I_{\text {emag }}=-10 \mathrm{~A}$

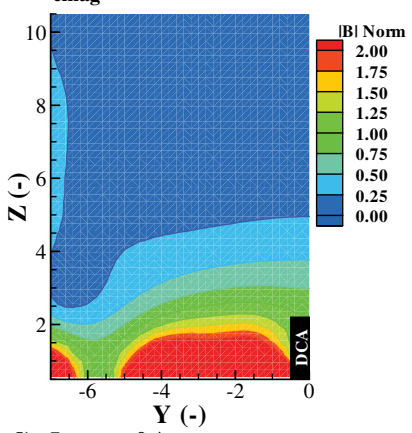

d) $I_{\text {emag }}=\mathbf{0 A}$
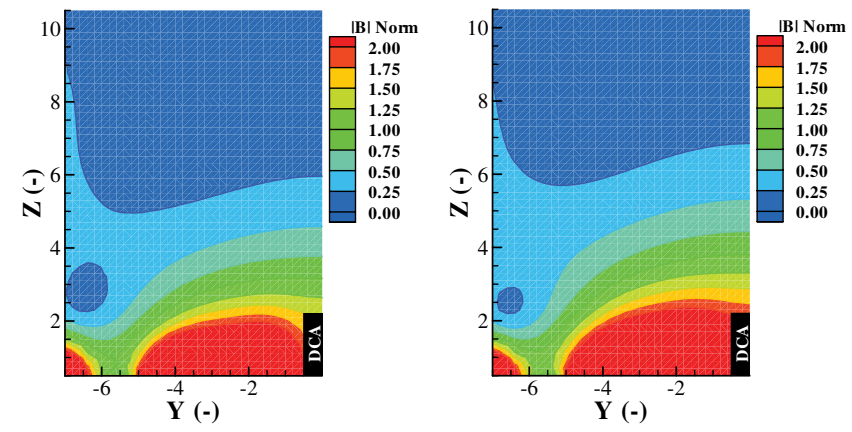

f) $I_{\text {emag }}=+10 \mathrm{~A}$

e) $I_{\text {emag }}=+5 \mathrm{~A}$

Fig. 1 Investigated magnetic field configurations (plots correspond to $X=0 \mathrm{~cm}$ plane based on Fig. 2).

at $0 \mathrm{~A}$ ) and four magnetic iron c-channels to each of the anode walls. Placing the magnetic iron c-channels at the cusp locations increases the strength of the intercusp field and effectively encloses the $50 \mathrm{G}$ contour. By definition the $50 \mathrm{G}$ contour is considered enclosed if it does not intersect with the anode walls or backplate. The seventh configuration was asymmetric with an increase in magnetic field strength near the off-centerline DCA. This configuration may force the peak current density location to shift from directly downstream of the off-centerline DCA to the centerline DCA location.

Six of the investigated magnetic field configurations are shown in Fig. 1. Each plot shows half of the $X=0 \mathrm{~cm} 2$-D plane based on the coordinate system shown in Fig. 2. Spatial dimensions are normalized by the NEXT DCA keeper diameter and magnetic field

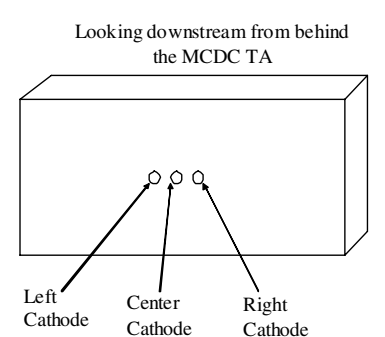

a)

Fig. 2 MCDC coordinate system a) looking downstream and b) looking

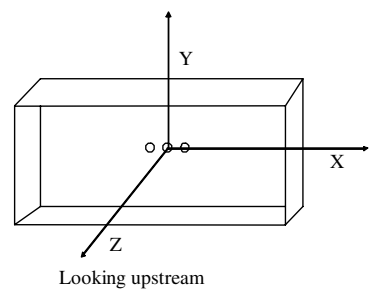

b) upstream. 


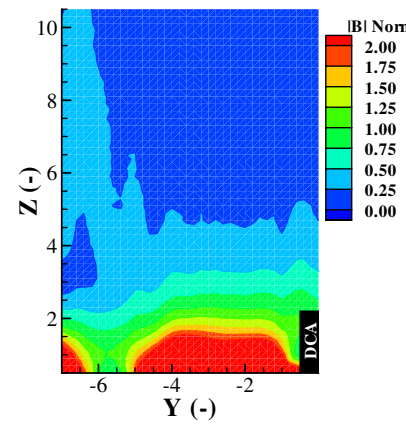

a) Experimentally measured

Fig. 3 Comparison of magnetic fields for the $X=0 \mathrm{~cm}$ plane with $I_{\text {emag }}=0$ A.

values are normalized by the center DCA keeper exit-plane magnetic field strength for the $0 \mathrm{~A}$ electromagnet configuration. This allows different magnetic field configurations to be compared with the normalized dimensions. A notional DCA is also shown.

\section{Comparison of Experimental and Simulated Magnetic Fields}

The designed MCDC magnetic field topology was validated by comparing 2-D experimental magnetic field maps with the simulation data over six planes for six magnetic field configurations. Excellent agreement was obtained between the experimentally measured profiles and the MagNet ${ }^{\mathrm{TM}}$ simulations. At spatial locations near the permanent magnets, an average percent difference of approximately $\pm 15 \%$ was obtained. Furthermore, the experimental maps verify that each DCA is located in a similar magnetic field environment. Comparisons of the all-permanentmagnet and five electromagnet configurations showed that a $-5 \mathrm{~A}$ electromagnet current is required to recover the all-permanentmagnet case. Again, this result is due to the presence of magnetic iron material, which increases the magnetic field even without electromagnet current. Furthermore, this result also suggests that it may be possible to recover any all-permanent-magnet magnetic field from the electromagnet augmented configuration. Finally, verification of the enclosure of the $50 \mathrm{G}$ contour was validated. Figure 3 shows a comparison of the simulated and experimentally measured magnetic field for the 0 A electromagnet configuration.

\section{Experimental Apparatus and Procedures}

\section{A. MCDC Test Article}

A coordinate system was constructed such that the center DCA opening in the backplate of the MCDC is considered the origin. Looking downstream from behind the MCDC, the positive $\mathrm{Z}$-axis extends in the downstream direction, the positive $\mathrm{X}$-axis extends to the left, and the positive Y-axis extends in the upward direction. A graphical representation of the coordinate system is shown in Fig. 2.

The MCDC was operated inside the large vacuum test facility [16] for all experiments and corrected operating pressures were below $4.0 \times 10^{-6}$ torr on xenon. The electromagnet was mounted to the MCDC backplate for all experiments. Those experiments requiring enclosure of the $50 \mathrm{G}$ contour also used the magnetic iron c-channels mounted to the anode walls.

Two dormant cathode units (DCUs) were mounted at two of the cathode locations. Each DCU was designed and constructed as similar to the active DCA as possible. Each DCU has a copper cathode tube with a chamfered orifice and a surrounding copper keeper tube with an orifice. Each DCU was also connected to a propellant feed system through a propellant isolator. This allowed the effect of propellant flow through the DCUs on test article (TA) performance and grid-plane uniformity to be studied. A photograph of a DCU is shown in Fig. 4.

Discharge chamber performance was investigated, so the MCDC was operated as a simulated ion thruster without beam extraction [11]. Brophy has shown that simulated operation accurately characterizes discharge chamber performance when the neutral

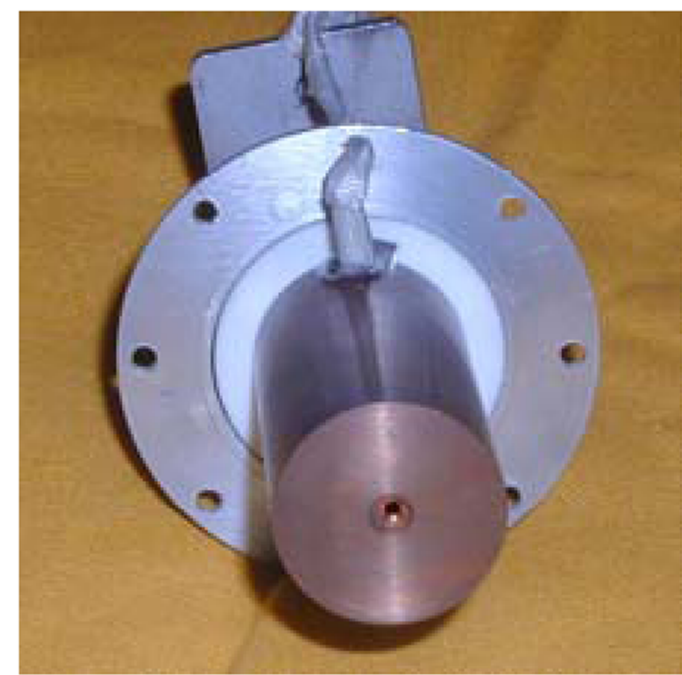

Fig. 4 Photograph of a DCU.

density is maintained equivalent to beam-extraction operation. However, plasma properties, such as the electron temperature, have been shown to vary depending on simulated or beam-extraction operation [11]. An ion collection grid was mounted at the ion extraction plane; high-voltage ion optics were not required. The ion collection grid was constructed of nonmagnetic stainless steel with holes arranged in a staggered pattern over the active area of the grid. The open area fraction of the ion collection grid was measured to be similar to the HiPEP ion optics open area fraction [12]. Simulated operation is typically performed using the ion optics as the ion collection grid. For this investigation, optics were unavailable, so a grid with similar open area fraction was used to maintain a similar neutral loss rate.

A reverse-feed plenum was designed based on the NEXT propellant feed system. This type of configuration has been shown to increase ion engine performance [17]. A NEXT hollow cathode with a surrounding keeper was used as the DCA. Only one DCA was used for all experiments presented here. The DCA was simply moved from the center to the left position for off-centerline, left DCA operation. The final thruster assembly consisting of the ion collection grid, NEXT DCA, DCUs, plenum, and MCDC is referred to as the MCDC test article (TA), or simply, TA.

Electrically the TA was set up for simulated ion thruster operation described by Brophy [11]. An engine bias supply raised the TA cathode common $25 \mathrm{~V}$ above facility ground, thus preventing electrons from leaving the TA and allowing for the extracted current to be measured. The collection grid was biased $-20 \mathrm{~V}$ with respect to the cathode to measure the ion current to the grid. During TA operation, the DCA was always connected to the circuit and the DCUs were connected or isolated from the circuit through switches. The three keepers were connected to the anode through a $10 \mathrm{kOhm}$ resistor. The electromagnet was electrically isolated from the TA. A schematic of the electrical circuit is shown in Fig. 5.

\section{B. Grid-Plane Probes}

An approximation of the grid-plane current density distribution was obtained by using 13 button probes placed at multiple locations on the ion collection grid. Each button probe consisted of a $0.32-\mathrm{cm}-$ diam stainless steel rod flush-mounted inside an alumina tube. The button probes are essentially planar Langmuir probes except the bias voltage is no longer adjusted but held constant in the ion saturation regime. For the experiments presented here, the button probes were biased $-20 \mathrm{~V}$ with respect to cathode common. Collected current was calculated by measuring the voltage drop across a current shunt and dividing by the known resistance $(10 \mathrm{kOhm})$. A schematic of the probes and their electrical setup is shown in Fig. 6. The location of the button probes on the grid is schematically depicted in Fig. 7. The probe locations on the extraction grid, as referenced to the coordinate system in Fig. 2, are presented in Table 1. 


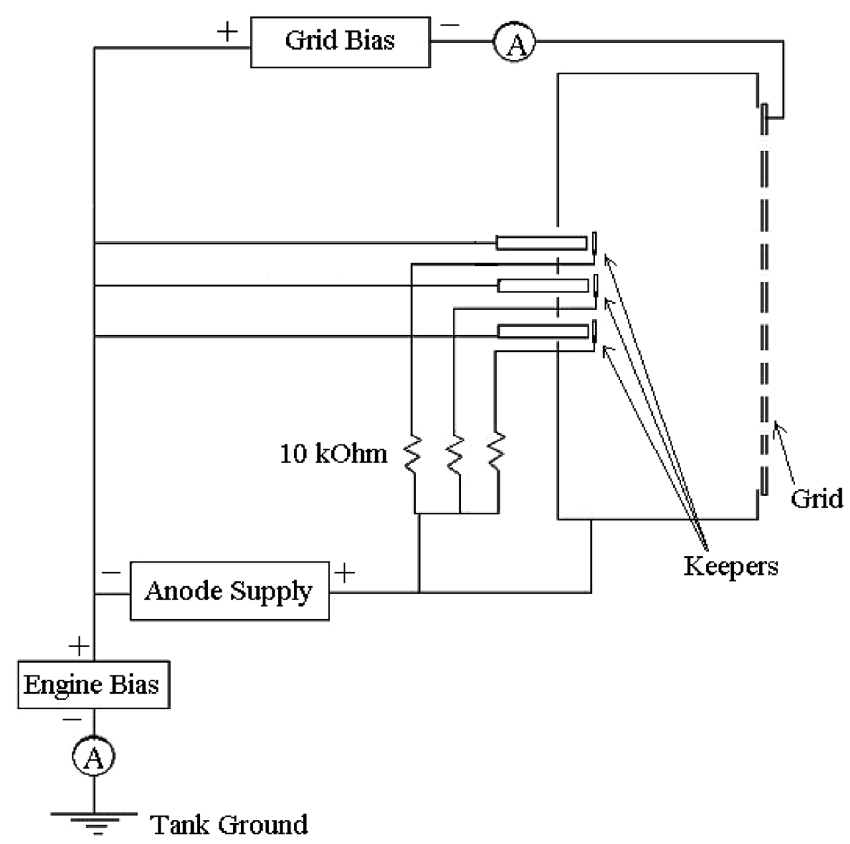

Fig. 5 Schematic of the MCDC TA electrical circuit.

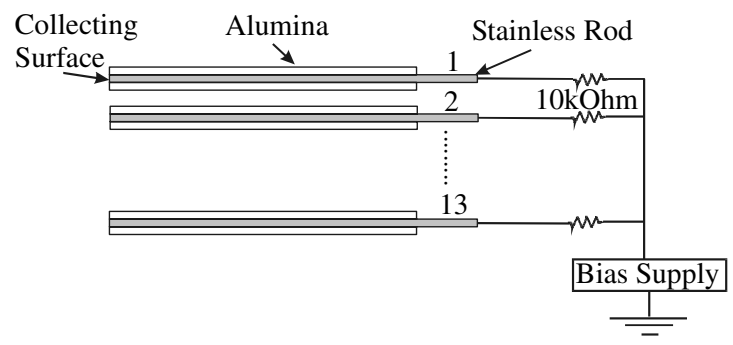

Fig. 6 Schematic of the grid-plane button probes.

\section{Data Acquisition Systems}

TA operating parameters and the button probe currents were recorded using an Agilent data logger with two 20-channel multiplexers. The data logger recorded discharge current and voltage, active cathode and keeper current, collection grid bias voltage and current, cathode common bias voltage and extracted current, cathode heater voltage and current, electromagnet voltage and current, and the button probe currents. Depending on the DCU connectivity, the data logger also recorded either DCU cathode and keeper current or DCU cathode and keeper floating voltage. In the case where a current was recorded, the voltage across a current shunt was measured and then divided by the known resistance. With this setup, real-time performance data were obtained and data collection was extremely time efficient. One sweep through all used channels required approximately $1.5 \mathrm{~s}$.

A Tektronix TDS 3034B oscilloscope was used to measure discharge voltage oscillations. Reported peak-to-peak voltage oscillations are the difference between maximum and minimum values recorded during a $4 \mathrm{~ms}$ oscilloscope trace sweep. Typical voltage oscillations for operating conditions reported here have peak-to-peak oscillations less than $1.5 \mathrm{~V}$, showing that the cathode was operating in the desired spot mode.

\section{Performance and Flatness Calculations}

Performance curves are generated by plotting ion production cost as a function of propellant utilization efficiency $[11,12,18]$. The following equations were used to determine the performance of the TA [11]:

$$
J_{g}=J_{s}+J_{b}
$$

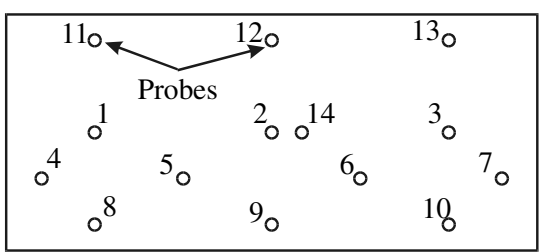

Looking upstream at the ion extraction area

Fig. 7 Schematic of the grid-plane button probes locations.

$$
\begin{gathered}
\hat{\phi}_{i}=\frac{0.35 J_{s}+J_{b}}{J_{g}} \\
\eta_{\text {ud }}=\frac{\phi_{i} J_{g}}{\hat{\dot{m}}+\phi_{i} J_{g}\left(1-\hat{\phi}_{i} / \phi_{i}\right)} \\
\varepsilon_{b}=\frac{\left(J_{d}-\phi_{i} J_{g}\right) V_{d}}{\phi_{i} J_{g}}
\end{gathered}
$$

The high-voltage ion optics transparency, $\phi_{i}$, was estimated at $70 \%$ [12]. The parameters $\varepsilon_{b}$ and $\eta_{\text {ud }}$ were calculated by setting $J_{d}$ and $\hat{\dot{m}}$, and then measuring $J_{s}, J_{b}$, and $V_{d}$. Performance curves were generated by incrementally decreasing $J_{d}$ from the nominal $30 \mathrm{~A}$ value and recording the new values. Unfortunately, increasing $J_{d}$ was not an option because the DCA has a maximum current rating of $30 \mathrm{~A}$.

The value of 0.35 in Eq. (2) represents the fraction of ions that strike the ion collection grid and subsequently exit the discharge chamber. Previous researchers have found this value to be closer to 0.50 or $0.55[11,18]$. Brophy has shown that discharge chamber performance can be characterized by simulated operation only if the neutral density remains equivalent to the beam-extraction condition [11]. In this case, the beam-extraction condition is assumed to be the HiPEP thruster because it has similar geometry and magnetic field structure to the TA [12]. An exiting ion fraction of 0.35 was calculated by equating the neutral density in the TA during simulated operation with the HiPEP thruster during beam extraction. This procedure was identical to that applied by Brophy to experimentally determine the simulated ion thruster flow rates [11].

Flatness is calculated as the average beam current density divided by the peak beam current density [19]. In this case, the current density was measured at discrete points at the grid-plane by the 13 grid-plane button probes. Flatness was calculated by averaging the ion current density of the 13 probes and then dividing by the maximum. Using the 13 grid-plane button probes to calculate flatness assumes that the probes were both uniformly distributed on the grid and that a sufficient number of probes were used. Table 1 gives the locations of the 13 grid-plane button probes. The bottom half of the grid contained 10 uniformly distributed probes, while the other three probes were located on the top half of the grid. The three probes on

Table 1 Button probe locations on the TA extraction grid

\begin{tabular}{lcc}
\hline \hline Grid-plane probe number & $X$ distance, $\mathrm{cm}$ & $Y$ distance, $\mathrm{cm}$ \\
\hline 1 & -29.2 & 0.0 \\
2 & 0.0 & 0.0 \\
3 & 29.2 & 0.0 \\
4 & -39.4 & -7.8 \\
5 & -13.7 & -7.8 \\
6 & 13.7 & -7.8 \\
7 & 39.4 & -7.8 \\
8 & -29.2 & -15.7 \\
9 & 0.0 & -15.7 \\
10 & 29.2 & -15.7 \\
11 & -29.2 & 15.7 \\
12 & 0.0 & 15.7 \\
13 & 29.2 & 15.7 \\
$14-$ Langmuir probe & 5.2 & 0.0 \\
\hline \hline
\end{tabular}


the top half of the grid validated the grid-plane current density symmetry about the MCDC midplane. A sufficient number of probes were used because the average current density obtained by the probes showed good agreement with the grid-plane current density, calculated by dividing the collected grid current by the grid collection area.

\section{E. Simulated Operation Flow Rate Determination}

During simulated ion thruster operation, the DCA and main plenum mass flow rates are reduced to maintain the beam-extraction internal neutral density [11]. A relationship for the reduced flow rate based on the beam-extraction flow rate has been determined by Brophy [11]. However, the TA has never been operated with beam extraction, so a reduced flow rate cannot be calculated. Therefore, TA mass flow rates for simulated ion thruster operation were experimentally determined by monitoring the grid-plane ion current as a function of flow rate. This procedure was identical to that applied by Brophy to experimentally determine the simulated ion thruster flow rates [11]. The flow rate corresponding with the maximum gridplane ion current was assumed to be the reduced flow rate. These experiments were conducted for both centerline and off-centerline DCA positions and for multiple magnetic field configurations. Measurement of performance parameters as a function of time showed that a wait time of approximately 2 min was required for the flow rate adjustment to equilibrate inside the TA.

Initially, the DCA and main plenum flow rates were set at the HiPEP beam-extraction level [12]. First, the main plenum flow was held constant while the DCA flow was reduced and grid-plane current was measured. Next, the same procedure was completed with the DCA flow held constant while the main plenum flow was adjusted. As flow rate decreased, discharge voltage oscillations increased. Large voltage oscillations occur when the cathode operates in "plume mode," which can be detrimental to cathode life [20]. Previous researchers have suggested that the preferred "spotmode" operation is defined by peak-to-peak voltage oscillations less than $5 \mathrm{~V}[4,5]$. The DCA and main plenum flow rates that provide the largest grid-plane current and relatively low peak-to-peak discharge voltage oscillations are considered the reduced, simulated ion thruster flow rates for the TA. The DCA and main plenum flow rates for all investigated TA configurations are summarized in Table 2. For the configuration with the electromagnet at $-5 \mathrm{~A}$, the peak-to-peak discharge voltage oscillations increase significantly above $5 \mathrm{~V}$ and therefore data were not collected at this condition. More information regarding this configuration is provided later in this paper.

\section{Results and Analysis}

A stable, spot-mode discharge was successfully obtained for all magnetic field configurations investigated. Unfortunately, to operate in spot-mode, the -5 A electromagnet configuration required an $8 \%$ larger DCA flow rate than the other configurations. At the elevated flow rate, ion production costs and propellant utilization efficiencies were $\sim 125 \%$ larger and $\sim 38 \%$ less than any of the other

Table 2 Results of the reduced flow rate study for all DCA and magnetic field configurations investigated

\begin{tabular}{lccc}
\hline \hline DCA activity & Magnetic field configuration & DCA (sccm) & Main $(\mathrm{sccm})$ \\
\hline Left active & 50 G enclosed & 5.73 & 24.8 \\
& Asymmetric & 6.12 & 30.9 \\
& Electromagnet -10 A & 6.12 & 30.9 \\
& Electromagnet -5 A & N/A & N/A \\
& Electromagnet 0 A & 6.12 & 30.9 \\
& Electromagnet +5 A & 6.12 & 30.9 \\
Center active & Electromagnet +10 A & 6.12 & 30.9 \\
& 50 G enclosed & 5.73 & 24.8 \\
& Electromagnet -10 A & 5.73 & 30.9 \\
& Electromagnet -5 A & N/A & N/A \\
& Electromagnet 0 A & 5.73 & 30.9 \\
& Electromagnet +5 A & 5.73 & 30.9 \\
& Electromagnet +10 A & 5.73 & 30.9 \\
\hline \hline
\end{tabular}

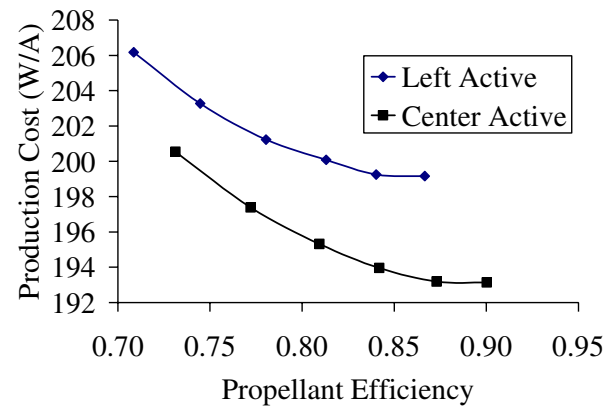

Fig. 8 TA performance curves for the $0 \mathrm{~A}$ electromagnet configuration.

configurations, respectively. As a result, the -5 A configuration was not further investigated.

The asymmetric magnetic field configuration was investigated to determine if increasing the magnetic field on the left side of the TA during left DCA operation causes the grid-plane current peak location to shift. Results do not show a shift in the peak current density location for the asymmetric magnetic field configuration. The shift in the peak may be too small for the button probe distribution to resolve or the magnetic field may not be strong enough to cause a substantial shift in location.

\section{A. Performance}

Characteristic performance curves for the TA are shown in Fig. 8. The curves shown are for center and left active DCA configurations, which have discharge voltages of 27.5 and $26.8 \mathrm{~V}$, respectively, for the nominal 30 A discharge current.

TA performance curves show an increase in ion production cost for decreasing propellant utilization efficiency. This same trend has been documented by Sovey [21] and Patterson [22] for a divergent field thruster and a 30-cm ring-cusp ion thruster, respectively, and it has also been reported for the HiPEP ion thruster [12]. Operation with the DCA on centerline and off centerline (left) show identical trends. Ion production costs are larger when the DCA is active at the off-centerline, left position. These trends are similar for all magnetic field configurations investigated.

Magnetic field configuration has a significant effect on discharge chamber performance. Ion production cost and propellant utilization efficiency as a function of magnetic field configuration for the nominal 30 A discharge with the center DCA active is shown in Fig. 9.

Propellant efficiency increases with backplate magnetic field strength, whereas ion production cost decreases. As the backplate magnetic field increases with electromagnet current, the grid-plane ion current increases. This trend has also been documented by Sovey [21] and Patterson [22] for a 30-cm ring-cusp discharge chamber. The +10 A electromagnet configuration provides the lowest production costs with the highest propellant uses, followed by the $+5 \mathrm{~A}$ and $0 \mathrm{~A}$ configurations, respectively. Although the $50 \mathrm{G}$ enclosed configuration increases the magnetic field along the anode walls of the TA, this does not decrease production costs. Performance numbers for other ion thruster discharge chambers can be found in Table 3.

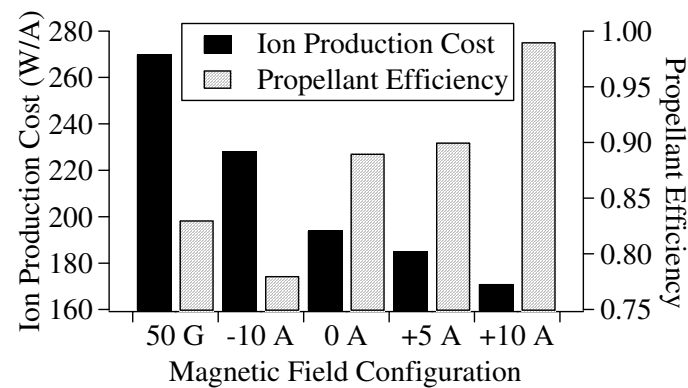

Fig. 9 Ion production cost and propellant utilization efficiency for investigated magnetic field configurations. 


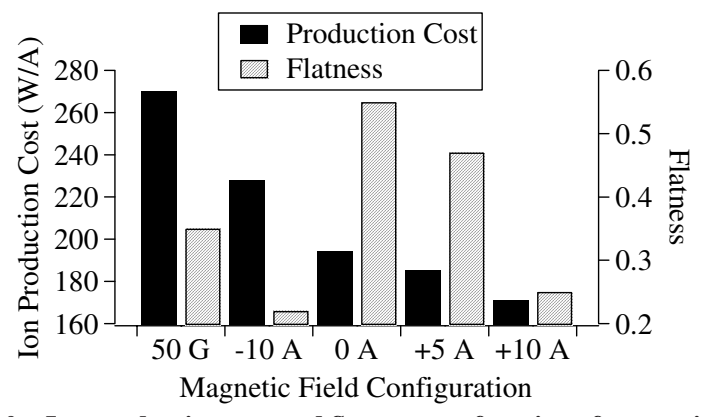

Fig. 10 Ion production cost and flatness as a function of magnetic field configuration.

\section{B. Uniformity and Flatness}

The maximum grid-plane current density is always directly downstream of the DCA. When the off-centerline, left DCA is active, the peak current density location shifts to the left side of the TA, directly downstream of the DCA. Magnetic field configuration has a significant impact on the flatness of the grid-plane ion current. As the backplate magnetic field increases, the measured flatness increases and then subsequently decreases, reaching a value of 0.55 for the $0 \mathrm{~A}$ electromagnet configuration with the center DCA active. This trend is illustrated in Fig. 10.

Sovey's investigation found that increasing the backplate magnetic field reduced the flatness [21]. This trend is also exhibited by the TA results, which show that increasing the upstream magnetic field too much can cause the flatness to decrease. Overall, these results suggest that when designing the magnetic field of a rectangular discharge chamber, there is a tradeoff between ion production cost, propellant utilization efficiency, and flatness. Compared with contemporary discharge chambers, flatness for all magnetic field configurations except the $0 \mathrm{~A}$ and $+5 \mathrm{~A}$ configurations is low. A comparison of TA flatness at the $0 \mathrm{~A}$ electromagnet configuration with other contemporary ion thruster discharge chambers is shown in Table 3.

\section{DCU Connectivity and Flow Rate}

The DCUs were operated either electrically connected or isolated from the electrical circuit while their flow rate was set at zero, half the DCA flow, and the full DCA flow rate. Total flow rate was kept constant during this procedure, so the main plenum flow rate was reduced as the DCU flow rate was increased. Discharge voltage was found to vary a maximum of $1 \mathrm{~V}$ as DCU flow increased. Ion production cost and propellant utilization efficiency did not change with DCU flow rate and electrical connectivity. Flatness improved as DCU flow rate increased for all configurations except the $50 \mathrm{G}$ enclosed configuration. However, most improvements were only on the order of $2-3 \%$, with a maximum $10 \%$ increase for the $0 \mathrm{~A}$ configuration with the left DCA operational.

Figure 11 shows the effects of flow rate on the DCU keeper floating voltage and collected current. When the DCUs are isolated from the TA electrical circuit, an increase in DCU flow rate causes the floating voltage of both the cathode and keeper to increase. DCU floating voltages are slightly larger than the active DCA keeper floating voltage, which is typically $5-7 \mathrm{~V}$. When electrically connected to the TA, the DCU cathode and keepers collect current.

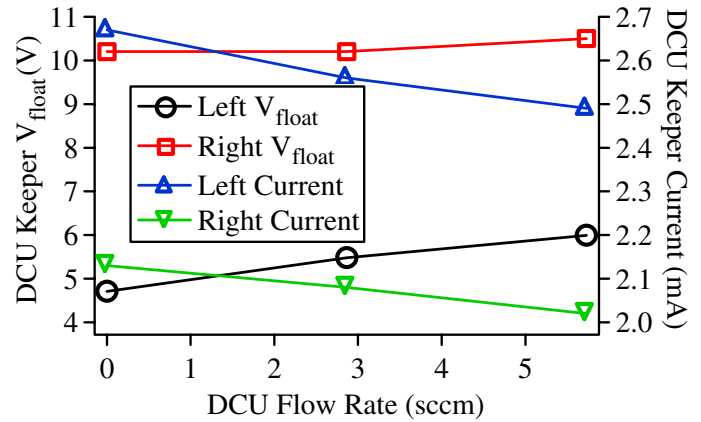

a) Middle DCA active

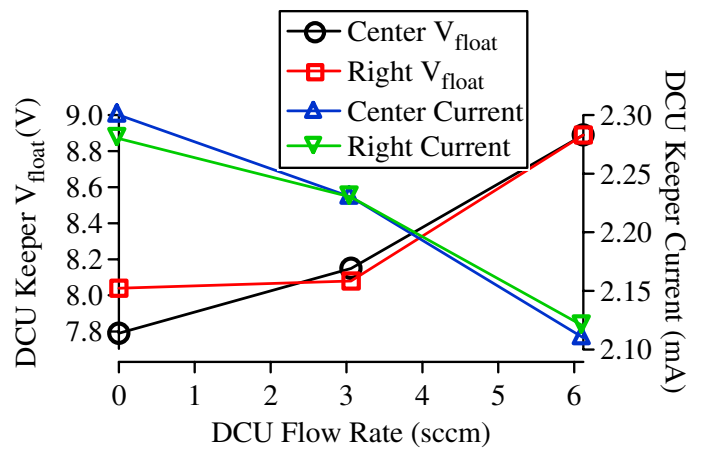

b) Left DCA active

Fig. 11 DCU keeper currents and floating voltages for the 0 A electromagnet configuration.

DCU keeper currents were similar to the active DCA keeper current, whereas DCU cathode currents were orders of magnitude less than the DCA cathode current. This is to be expected because the active DCA cathode was emitting the bulk of the discharge current through electron and ion production, whereas the DCUs were simply collecting a current. As DCU flow rate increases, DCU keeper current decreases, but cathode current does not display a consistent trend. A decrease in keeper current signifies a decrease in plasma bombardment of the keeper, which may lead to a decrease in keeper erosion. Furthermore, because the DCUs have larger floating voltages, bombarding ions gain less energy and therefore less erosion is expected to occur.

\section{Optimized TA Magnetic Field Configuration}

Overall, the 0 A electromagnet TA configuration is the optimized configuration. Flatness and performance were both considered when determining the optimum configuration. The $0 \mathrm{~A}$ configuration has a relatively high flatness, $0.55 \pm 0.02$ and $0.53 \pm 0.02$, and relatively low discharge losses, $194 \pm 6 \mathrm{~W} / \mathrm{A}$ at $0.89 \pm 0.03$ propellant efficiency and $199 \pm 6 \mathrm{~W} / \mathrm{A}$ at $0.87 \pm 0.03$ propellant efficiency, for both center and left DCA operation, respectively. Other configurations have lower production costs, but they also have decreased flatness. The optimum configuration does not enclose the $50 \mathrm{G}$ contour within the TA. For the nominal 30 A discharge current, the discharge voltage is $27.5 \mathrm{~V}$ with peak-to-peak oscillations less than $1 \mathrm{~V}$ and a simulated beam current of $3.78 \mathrm{~A}$.

Table 3 Comparison of contemporary ion thruster discharge chambers with the MCDC TA simulated operation for the 0 A electromagnet configuration

\begin{tabular}{|c|c|c|c|c|c|}
\hline Thruster & Geometry & Propellant efficiency & Ion production cost, $\mathrm{W} / \mathrm{A}$ or $\mathrm{eV} /$ ion & Flatness & Beam current, A \\
\hline NEXT $[19,25]$ & Cylindrical & 0.89 & 135 & 0.66 & 3.52 \\
\hline NEXIS [18] & Cylindrical & 0.90 & 160 & 0.85 & 3.90 \\
\hline MCDC TA (simulated) & Rectangular & 0.89 & 194 & 0.55 & 3.78 \\
\hline
\end{tabular}




\section{Conclusion}

A multiple-cathode, rectangular ion thruster discharge chamber can be operated with a stable, spot-mode discharge with the DCA located at centerline and off-centerline positions. The thruster magnetic field configuration has profound influences on the performance and flatness of the device. Specifically, there is a tradeoff between ion production cost, propellant utilization efficiency, and flatness when optimizing the magnetic field. Larger near-DCA magnetic field strengths decrease production costs and increase efficiency, but also decrease flatness. The MCDC investigated should be operated with a 0 A electromagnet current. Furthermore, results suggest that the MCDC can be operated with performance and flatness parameters similar to other state-of-the-art ion thruster discharge chambers.

The electrical connectivity and flow rate of the DCUs do not significantly affect production cost, efficiency, or flatness. Therefore, the DCUs can be operated in any configuration without affecting thruster operation. Expelling propellant through the DCUs does not degrade thruster performance because the main plenum flow rate is reduced such that the total flow rate remains constant. Furthermore, the DCUs are expected to suffer less ion bombardment erosion than the active DCA because the DCU keeper floating voltage is larger and DCU propellant flow rate decreases bombarding ion current. Further detailed investigation of DCU ion bombardment erosion is required, but results presented here indicate that a MCDC is a viable propulsion candidate that should be considered for future long-life, high-throughput missions that wish to use flight qualified or flight heritage DCA designs.

\section{Acknowledgments}

We would like to thank Michael Patterson of NASA John H. Glenn Research Center (GRC) for the financial support of this research through research grant NNC04GA67G and for use of government equipment. We would like to acknowledge John Foster (grant monitor) who has been the principal contact at NASA GRC. J. L. Rovey was also supported through a Michigan Space Grant Consortium graduate fellowship. We would also like to thank the entire research group at the Plasmadynamics and Electric Propulsion Laboratory who have been instrumental in this investigation, Daniel Herman for his assistance with the NEXT discharge cathode and the initial operation of the test article, Bailo Ngom for assistance with hardware construction, and Terry Larrow for fabricating the hardware used in this study. This support is gratefully acknowledged.

\section{References}

[1] Patterson, M. J., Roman, R. F., and Foster, J. E., "Ion Engine Development for Interstellar Precursor Missions," AIAA Paper 20003811, July 2000.

[2] Oleson, S., "Electric Propulsion Technology Development for the Jupiter Icy Moon Orbiter Project," AIAA Paper 2004-3449, July 2004.

[3] Rawlin, V. K., Williams, G. J., Pinero, L., and Roman, R. F., "Status of Ion Engine Development for High Power, High Specific Impulse Missions," IEPC Paper 01-096, Oct. 2001.

[4] Sengupta, A., Brophy, J. R., Anderson, J. R., Garner, C. E., de Groh, K., Karniotis, T., and Banks, B., "An Overview of the Results from the 30,000 Hr Life Test of Deep Space 1 Flight Spare Ion Engine," AIAA
Paper 2004-3608, July 2004.

[5] Sengupta, A., Brophy, J. R., and Goodfellow, K., "Status of the Extended Life Test of the Deep Space 1 Flight Spare Engine After 30,352 Hours of Operation," AIAA Paper 2003-4558, July 2003.

[6] Sengupta, A., "Destructive Physical Analysis of Hollow Cathodes from the Deep Space 1 Flight Spare Ion Engine 30,000 Hr Life Test," IEPC Paper 2005-026, Oct.-Nov. 2005.

[7] Kamhawi, H., Soulas, G. C., Patterson, M. J., and Frandina, M. M., "NEXT Ion Engine 2000 Hr Wear Test Plume and Erosion Results," AIAA Paper 2004-3792, July 2004.

[8] Williams, G. J., Hickman, T. A., Haag, T. W., Foster, J. E., and Patterson, M. J., "Preliminary Wear Analysis Following a 2000-h Wear Test of the HiPEP Ion Thruster," IEPC Paper 2005-260, Oct.Nov. 2005.

[9] Snyder, J. S., Goebel, D. M., Polk, J. E., Schneider, A. C., and Sengupta, A., "Results of a 2000-Hour Wear Test of the NEXIS Ion Engine," IEPC Paper 2005-281, Oct.-Nov. 2005.

[10] Kovaleski, S. D., Patterson, M. J., Soulas, G. C., and Verhey, T. R., "A Review of Hollow Cathode Testing for the International Space Station Plasma Contactor," IEPC Paper 01-271, Oct. 2001.

[11] Brophy, J. R., "Simulated Ion Thruster Operation Without Beam Extraction," AIAA Paper 90-2655, July 1990.

[12] Foster, J. E., Haag, T. W., Kamhawi, H., Patterson, M. J., Malone, S., Elliott, F., Williams, G. J., Sovey, J. S., and Carpenter, C., "The High Power Electric Propulsion Thruster," AIAA Paper 2004-3812, July 2004.

[13] MagNet 6.0, Infolytica Corporation, Montréal, 2004, http:// www.infolytica.com [cited 18 July 2006].

[14] Chen, F. F., Introduction to Plasma Physics and Controlled Fusion, Vol. 1, Plasma Physics, Plenum, New York, 1984.

[15] Nicholson, D. R., Introduction to Plasma Theory, John Wiley \& Sons, New York, 1983.

[16] Rovey, J. L., and Gallimore, A. D., "Dormant Cathode Plasma Properties and Erosion Analysis in a Multiple-Cathode, High-Power, Rectangular Discharge Chamber," AIAA Paper 2005-4241, July 2005.

[17] Reader, P. D., "Experimental Effects of Propellant Introduction Mode on Electron-Bombardment Ion Rocket Performance," NASA TN D2587, 1965.

[18] Goebel, D. M., Polk, J. E., and Sengupta, A., "Discharge Chamber Performance of the NEXIS Ion Thruster," AIAA Paper 2004-3813, July 2004.

[19] Soulas, G. C., Haag, T. W., and Patterson, M. J., "Performance Evaluation of $40 \mathrm{~cm}$ Ion Optics for the NEXT Ion Engine," AIAA Paper 2002-3834, July 2002.

[20] Domonkos, M. T., "Evaluation of Low-Current Orificed Hollow Cathodes," Doctoral Thesis, Dept. of Aerospace Engineering, Univ. of Michigan, Ann Arbor, MI, 1999.

[21] Sovey, J. S., "Improved Ion Containment Using a Ring-Cusp Ion Thruster," Journal of Spacecraft and Rockets, Vol. 21, No. 5, Sept.Oct. 1984, pp. 488-495.

[22] Patterson, M. J., "Performance Characteristics of Ring-Cusp Thrusters with Xenon Propellant,” AIAA Paper 86-1392, June 1986.

[23] Soulas, G. C., "Performance Evaluation of Titanium Ion Optics for the NASA $30 \mathrm{~cm}$ Ion Thruster," IEPC Paper 01-092, Oct. 2001.

[24] Polk, J. E., Anderson, J. R., Brophy, J. R., Rawlin, V. K., Patterson, M. J., Sovey, J. S., and Hamley, J., "An Overview of the Results from an 8200 Hour Wear Test of the NSTAR Ion Thruster," AIAA Paper 992446, June 1999.

[25] Soulas, G. C., Kamhawi, H., Patterson, M. J., Britton, M. A., and Frandina, M. M., "NEXT Ion Engine 2000 Hr Wear Test Results," AIAA Paper 2004-3791, July 2004.

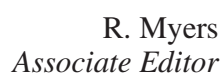

